\title{
Neighbourhood level versus individual level correlates of women's body dissatisfaction: toward a multilevel understanding of the role of affluence
}

\section{McLaren, L Gauvin}

See end of article for authors' affiliations

Correspondence to: Lindsay McLaren, GRIS, University of Montreal,' $\mathrm{PO}$ Box 6128 Downtown Station, Montreal, QC, Canada, H3C 3J7:

lindsaymclaren@hotmail.com

Accepted for publication 3 August 2001

\begin{abstract}
Study objective: This study examined the prevalence of body dissatisfaction as a function of individual level and neighbourhood level indicators of affluence.

Participants and setting: A subset of data from a larger random digit dialling telephone survey was used to obtain individual level data on body dissatisfaction, body weight and height, and income from a group of 895 adult women (age 24-56, 61\% English speaking) living in 52 neighbourhoods (census tract areas) within the provinces of Alberta, Ontario, and Quebec, Canada who were selected for their heterogeneity in social class.

Design: Aggregated census tract data from 1996 were used to develop neighbourhood indicators of affluence. Using hierarchical linear modelling, body dissatisfaction (dichotomous) was examined as a function of individual body mass index, individual level affluence and neighbourhood level affluence. Main results: The impact of body mass index on body dissatisfaction depended on the level of neighbourhood affluence: an average body mass index was associated with higher likelihood of reporting body dissatisfaction in a neighbourhood of above average affluence $(71 \%$ probability) than in a neighbourhood of average affluence $(58 \%$ probability), independent of a woman's individual affluence (whether she was low income or not).

Conclusion: It is concluded that a clearer understanding of the role of affluence on body dissatisfaction can be achieved by a joint examination of individual and neighbourhood level influences.
\end{abstract}

$\mathrm{T}$ he eating disorders and related symptoms (body dissatisfaction, dietary restraint) are commonly believed to be socially stratified such that higher prevalence of these phenomena are found among women of higher social class. However, empirical research on this position has been mixed. ${ }^{1}$ A notable feature of this literature is that social class is almost always measured as an individual level attribute. In this paper, we take the position that the inconsistent findings regarding the role of social class reflects-in part-the failure of extant research to consider the affluence of an individual's local environment, in addition to her personal level of wealth.

In considering the determinants of health outcomes in general, there is growing evidence that an individual's local environment or neighbourhood is an important source to consider. ${ }^{2}$ In particular, economic features of the neighbourhood have been associated with individual health phenomena including smoking, ${ }^{3}$ physical activity, ${ }^{4}$ and body weight. ${ }^{5}$ This neighbourhood level approach has been less evident in the psychological literature, and to our knowledge is non-existent in the literature on body dissatisfaction. Thus, in this study we introduce a neighbourhood level and individual level approach to the study of the relation between affluence and body dissatisfaction. The role of body dissatisfaction as a risk factor in the eating disorders underlies a substantial literature on this topic; however, the focus of this paper is body dissatisfaction in its own right.

\section{BACKGROUND}

Body dissatisfaction: A public health problem?

Body dissatisfaction is commonly reported by women in industrialised countries. Across women ranging in age from adolescence to midlife, research has consistently demonstrated that a large proportion if not a majority of women in any given sample report being dissatisfied with their bodies. $^{6-8}$
Furthermore, this dissatisfaction seems to carry notable consequences for health and wellbeing among these women. It has been linked with greater depressive affect, lower overall quality of life, fewer pleasant feelings (energetic, happy, upbeat), and more unpleasant feelings (for example, fatigued, tired, worn out ${ }^{9}$ among adult women. Also among adult women, body dissatisfaction has been associated with diminished sexual interest and sexual activity, less enjoyment of sex, ${ }^{10}$ and with marital dissatisfaction, independent of the woman's body size. ${ }^{11}$

Body dissatisfaction may also adversely influence health behaviours. It may prevent participation in physical activity, ${ }^{12}$ because a woman who is dissatisfied with her body may be reluctant to exercise in public and/or for which she must wear revealing clothing (for example, swimming). In addition, as smoking cessation is linked with weight gain, body dissatisfaction may be one reason why women are reluctant to stop cigarette smoking ${ }^{13}$ despite the far more negative health consequences of failure to stop. Among middle aged women, body dissatisfaction may also influence medical decisions at menopause. Women have cited perceived cosmetic benefits and anti-aging effects of hormone replacement therapy as reasons for choosing or not choosing this therapeutic option at menopause..$^{14}$ Finally, body dissatisfaction is linked with unhealthy weight loss tactics and disordered eating among women of various age groups.?

The widespread nature of body dissatisfaction, coupled with its consequences for health and wellbeing, render this topic an important one for further investigation. As there is substantial overlap among the body dissatisfaction and eating disorder literatures, we drew from research in both areas to create an appropriate empirical and conceptual backdrop for this research. 


\section{Body dissatisfaction and the role of affluence}

A common stereotype about eating disorders and related symptoms such as body dissatisfaction is that they are more prevalent in women of higher social class, although empirical findings on this relation have been mixed..$^{16}$ Some research has supported the existence of a positive gradient between socioeconomic status and disordered eating symptoms. ${ }^{17-20}$ Other studies have demonstrated an inverse relation, ${ }^{21}{ }^{22}$ or no relation at all. ${ }^{123-25}$

As a backdrop to these contradictory findings, eating disorders are quite clearly "illnesses of the affluent" when viewed on a global scale: they are most prevalent in Western, industrialised countries, ${ }^{26}$ which by definition are characterised by relative wealth. Despite reports of eating disorders and symptoms outside of Western cultures, ${ }^{27}$ these phenomena none the less remain far more common in countries of relative affluence. On the basis of this global social distribution, researchers have identified features of these "wealthy" environments that are linked with eating disorders; most notably, the thin ideal body shape held for women. Within Western societies, the existence of eating disorders and body dissatisfaction seems to have coincided with an increasingly thin standard of beauty as indicated by the size of female models $^{28}$ who continue to be underweight by normative standards. ${ }^{29}$ Moreover, it has been documented that greater exposure to media portrayals of this thin ideal is linked with higher likelihood of eating disorder symptoms. ${ }^{30} 31$ Greater internalisation of these "standards" has likewise been linked with greater body dissatisfaction. ${ }^{32}$ Overall, it seems that body dissatisfaction is a feature of wealthier environments (and their concomitant sociocultural risk factors) on a global scale, but it is currently unclear whether they covary with individual wealth within these industrialised countries.

\section{A new focus: the local environment}

Interestingly, despite the interest in socioeconomic status as a salient feature of the eating disorders, research has remained limited to these two extremes of socioeconomic status: individual wealth on the one hand and global affluence on the other. There have been no investigations into the role of intermediate level affluence - that is, the wealth of an individual's immediate living environment (or neighbourhood) on body dissatisfaction. This is despite there being several lines of thinking pointing to the importance of this level of influence.

Firstly, broader sociocultural factors (that is, thin standard for women's bodily attractiveness) observed in wealthier countries may be filtered through, and thereby made more or less salient by, local environmental factors including individuals (family, peers, work colleagues), goods (fashion magazines, diet food products), and services (gyms, weight loss centres, particular clothing stores), and these factors probably covary with the affluence of the local environment. ${ }^{2}$ For example, it is conceivable that in a wealthier neighbourhood, fashion magazines and diet products are more readily available and affordable, work out facilities and fashionable clothing stores are more plentiful and popular, and perhaps individuals are generally more invested in their bodies and appearance. In such an environment there may emerge a local or "micro" culture in which appearance investment is the norm. The broader sociocultural emphasis on appearance is thereby accentuated in this particular local environment.

In addition, it is probable that individual income-the indicator of socioeconomic status typically used in existing studies-is gauged by an individual against the income or social class of his or her neighbours, rendering individual social class a relative term that depends upon the social class of the surrounding area. For example, a poor individual living in a poor environment probably has different experiences than a poor individual living in a wealthier environment. This shows that both individual and local environmental affluence are important to consider, and failure to include the latter may have contributed to inconsistencies evident in the research on affluence and eating disorder symptoms. We aim to clarify this inconsistency by considering, independently, the income of the woman and of her local environment.

Finally, current thinking on social determinants of health ${ }^{33}{ }^{34}$ suggests that an examination of neighbourhood or small area variations may increase understanding of pathways or mechanisms through which socioeconomic factors influence health. Unlike statewide or nationwide comparisons, neighbourhood level analyses highlight environmental features that are proximal to the individual, and that may thereby have particular relevance to the health of residents. In other words, social and physical aspects of the neighbourhood may be identifiable as proximate mediators of the relation between affluence and a given health outcome. To date, this neighbourhood level approach has been implicated primarily in population health studies of health behaviours and chronic illness. To our knowledge it has not been applied to largely psychological outcomes such as eating disorders and body dissatisfaction.

Thus, in this paper we wish to merge two perspectives: (a) body dissatisfaction, for which research is overwhelmingly psychological (and individualistic) in nature, and (b) neighbourhood level determinants of health (affluence in particular), which has emerged largely from research in population health and epidemiology. Towards this end, we reviewed research from the following three bodies of literature: (a) the social stratification of body size, (b) the viability of neighbourhood level determinants of individual health in general, and (c) the apparent importance of the local environment within the specific context of eating disorders, to which body dissatisfaction is related.

\section{Social stratification of body size}

Body size, arguably the strongest predictor of body dissatisfaction among women ${ }^{20}$ is clearly stratified by socioeconomic status within industrialised societies. An inverse relation between individual socioeconomic status and body mass index has been consistently documented among women in these societies. ${ }^{18} 35$ Importantly, as overweight and obesity become common among the lower class, a thin body is reinforced as a mark of affluence, or of social distinction. ${ }^{36}$ It follows, theoretically, that it is the wealthy who will aspire to be thin, and who in turn will be most distressed by a discrepancy between their own body and that of the ideal (that is, experience the most body dissatisfaction). This leads to the position that body dissatisfaction should show predictable social patterning, with body dissatisfaction being more prevalent among wealthier women. As mentioned above, this pattern has been demonstrated, ${ }^{19}{ }^{37}$ but contradictory findings also exist. ${ }^{21}{ }^{24}$

\section{Small area variations in health outcomes}

Increasingly, population health researchers are examining small area or neighbourhood variations in health outcomes, as well as features of the local environment that enhance or hinder the health of its members. ${ }^{33} 38$ This line of thinking emerged from evidence of geographical variation in health status, whereby groups of people living in different locales are found to differ reliably in health behaviours and outcomes including smoking rates, physical activity involvement, and cardiovascular illness. This research emerged against the backdrop of a "social determinants of health" model, which emphasises risk factors that are social, cultural, economic, and political in nature..$^{39}$ Much of this research has focused on socioeconomic features of the community or neighbourhood, and has generally indicated that poorer areas, and areas in which income is unevenly distributed, suffer the worst health consequences. ${ }^{34}$ Moreover, this impact of community or neighbourhood socioeconomic status on individual health is over and above the impact of individual socioeconomic status. ${ }^{2}$ 
Of relevance to this paper, such small area variation has been documented in body size, with more disadvantaged neighbourhoods having a higher proportion of obese individuals as well as a higher average body mass index. ${ }^{5}$ This suggests that the affluence of one's local environment has an impact on body size. If these lower income areas are somehow more accepting of larger body sizes, and if the reverse is also true, that more affluent areas are more demanding of a thin body size, we can hypothesise that women's body dissatisfaction will show area patterning whereby body dissatisfaction is more prevalent in higher income neighbourhoods than in lower income neighbourhoods.

\section{Indirect evidence for small area variation in eating disorders and related symptoms}

Finally, some research in the eating disorder literature suggests that the notion of between neighbourhood differences may be particularly relevant to this topic. A unique feature of the eating disorder literature is the documentation of various "micro-environments" that are associated with, or that seem to facilitate, the emergence of eating disorder symptoms. Heightened prevalence of "drive for thinness", dietary restraint, and full blown eating disorders have been found within discrete, definable contexts such as ballet class, ${ }^{41-44}$ modelling schools, ${ }^{43}$ and private girls' schools. ${ }^{22}$ Such findings seem unique to the eating disorder literature; they are not evident in research on other health phenomena. Demonstrable differences in the prevalence of eating disorder symptoms between individuals within versus outside such environment highlight the importance of an individual's immediate environment in the development of these symptoms, and thus provides compelling rationale for the examination of the neighbourhood (that is, simply another type of immediate environment) as a determinant or risk factor for eating disorder symptoms.

\section{The current study}

In summary, there is much interest yet little consensus about the role of social class in the eating disorders and body dissatisfaction. Research on the clear social stratification of body size, the viability of neighbourhood level factors as determinants of health, and the importance of the local environment in the context of eating disorder symptoms, impelled us to examine the impact of neighbourhood level affluence on the body dissatisfaction of women residents. We believe that this topic holds some unique interest from the perspective of population health and social inequalities because, while existing research on neighbourhood health effects has focused on outcomes that are inversely related to socioeconomic status (higher socioeconomic status associated with better health outcome), the eating disorders and body dissatisfaction may show a unique reverse pattern, namely, higher socioeconomic status linked with worse outcome. Two questions are at the centre of this investigation. Firstly, is there between neighbourhood variation in body dissatisfaction? Secondly, to what extent can this variation be attributed to individual and neighbourhood affluence, respectively?

\section{METHODS \\ Participants}

The original sample, from which our sample was drawn, included over 3000 adult men and women age 24-56 who resided in 52 neighbourhoods defined by census tract boundaries ( selected from urban, suburban, and rural sites) in the provinces of Alberta, Ontario, and Quebec, Canada. All participants reported having lived in their particular neighbourhood for at least one year. For this study, we used data from a subset of 895 women: this data subset included all female participants for whom complete data were available on variables used in our analyses. * The majority of these women
(61\%) reported English as the language they typically speak at home, whereas 34\% reported usually speaking in French (4\% reported both French and English). The mean age of these women was 40.18 years (SD 9.73), and mean body mass index was $24.03 \mathrm{~kg} / \mathrm{m}^{2}$ ( SD 4.54). Just over half ( $\mathrm{n}=478,53.4 \%$ ) were "dissatisfied" with their body weight. The proportion of women who reported being "dissatisfied" increased with increasing body mass index category (categories based on Canadian standards from National Health and Welfare ${ }^{45}$ ): namely, $31 \%, 43 \%, 74 \%$, and $86 \%$ of "underweight" (body mass index <20), "acceptable weight" (body mass index 20-24.9), "some excess weight" (body mass index 25-27), and "overweight" (body mass index >27) women, respectively, reported body dissatisfaction. Average annual individual (family) income category was between $\$ 30000$ and $\$ 60000$, and $18.5 \%$ of these women were considered "low income" (reported annual family income of less than \$20 000).

\section{Characteristics of the small areas of interest}

In this study, a "neighbourhood" corresponds to a census tract. The 52 neighbourhoods were deliberately selected for their diversity in socioeconomic status, as follows. Within the three provinces, municipality defined regions were selected according to their degree of urbanisation (large urban, small urban, suburban, and rural). Within each of these categories, efforts were made to select census tracts that were high, medium, and low in affluence (defined in terms of average family and household income, as well as the percentage of low income families residing in the area). In most cases $(n=44)$, a municipality defined region encompassed several census tracts and therefore it was possible to select neighbourhoods (census tracts) of varying degrees of affluence within them. However, in two instances, a municipality defined region contained only a single census tract and therefore that single census tract was used in these cases. Furthermore, three rural regions were each divided into "village" to indicate residence in the commercial agglomeration of the region, and "township" to indicate residence in the surrounding areas, yielding the final six neighbourhoods for a total of 52. Although there is much debate over the conceptual and empirical definition of neighbourhood, ${ }^{33}$ we, like other researchers ${ }^{46}$ opted to use a census-based spatial definition in order to have access to systematically gathered economic information about our areas.

Across the 52 neighbourhoods used in this study, the mean neighbourhood income was \$61 280 and ranged from \$26 927 to $\$ 190068$. Average population size of the neighbourhoods was 4924 and ranged from 1118 to 12 973, with one outlying value of 44882 . The mean neighbourhood percentage of low income families was $20.3 \%$ and ranged from $1.7 \%$ to $62.7 \%$. The neighbourhoods with the highest average income were usually, but not always, the neighbourhoods with the lowest proportion of low income families. The mean age of women within the neighbourhoods ranged from 33.4 years to 46.8 years, with within neighbourhood standard deviations ranging from 5.52 years to 13.06 years

\section{Measures}

Body dissatisfaction

Body dissatisfaction was assessed using a single item: "are you happy with your current body weight or would you like to

*An independent samples $t$ test indicated that women with complete data on "income" and "body mass index" did not differ on "body

dissatisfaction" (our outcome variable) from those women with missing data on either or both of these two variables $(t(1627)=-0.861, p=0.71)$. Furthermore, a $\chi^{2}$ test indicated that patterns of missing data on body mass index $(n=167)$ and on body dissatisfaction $(n=22)$ were random across neighbourhoods $\left(\chi^{2}(52)=63.99, p=0.12\right.$ for body mass index; $\chi^{2}$ $(52)=33.98, p=0.98$ for body dissatisfaction). 
weigh more or less than you do?". Response options included "happy with current body weight", "would like to lose weight", "would like to gain weight", and "don't know/decline to respond". Among those who responded, responses were then re-coded to create a dichotomous variable: "satisfied" (happy with current body weight) versus "dissatisfied" (would like to lose or gain). $†$ Although the use of a single item for measurement may be limited in terms of psychometric evaluation, it is noteworthy that a one item assessment method is commonly used in population surveys that have assessed body dissatisfaction or desire to lose weight. ${ }^{87}$

\section{Individual level affluence}

Individual affluence was indicated by a dummy variable indicating "low income" or not. This information was based on the following question: "which of the following numbers best approximates your total family income over the past 12 months": "less than \$5000", “between \$5000 and \$10 000", "between \$10000 and \$15000", "between \$15000 and $\$ 20000 "$ ", "between \$20 000 and \$30 000", "between \$40 000 and $\$ 60000 "$ ", "over $\$ 60000 "$ and “don't know/decline to respond". Among the numerical responses, those who indicated "between \$15000 and \$20000" or lower were designated "low income". All others were designated "not low income".

\section{Neighbourhood level income}

Data on average family income for each neighbourhood were downloaded from Statistics Canada 1996 census tract databases. "Average family income" includes the average income of all census families, defined as families of married couples (with or without never married children), families of common law couples (with or without never married children), and lone parent families of either sex. Income refers to total money income received in 1995 by all family members 15 years and older. ${ }^{48}$

\section{Body mass index}

Body mass index $\left(\mathrm{kg} / \mathrm{m}^{2}\right)$ was calculated based on height and weight values provided by participants. Although self report values of height and weight are not without problems (women tend to underreport their weight and overestimate their height ${ }^{49}$ ), it is also generally true that self report and measured values of weight and height are quite highly correlated $\left(r>0.90\right.$ for adults $\left.{ }^{50}\right)$.

\section{Age}

Participants provided their age in years.

\section{Procedure}

Participants were recruited through random digit dialling procedures to participate in a 20 minute telephone interview on lifestyle and community resources. The data used in this study were a part of this larger research.

\section{Analytical strategy}

In light of our goal of disentangling the impact of individual level versus neighbourhood level affluence in the prediction of body dissatisfaction, we used hierarchical linear modelling analyses, which are appropriate for data in which one level of analysis (individuals, level 1) are nested within another (neighbourhoods, level 2). We followed Bryk and Raudenbush's ${ }^{51}$ model building procedure, as follows. Starting with the unconditional model (no predictor variables), we

†The majority of the "dissatisfied" women reported "wanting to lose weight" (93\%). We ran analyses with and without those who reported "wanting to gain weight" (7\%); results did not differ and thus results based on data from all women were retained. examined the amount of variance in body dissatisfaction (the outcome variable) that could be attributed to the individual and neighbourhood levels of analysis. Next, we entered level 1 predictors: age, body mass index, and individual affluence. These were entered one at a time as random effects; if a significant variance component was detected, the predictor was retained as a random effect; if not, the predictor was constrained to be fixed across neighbourhoods. Finally, we entered the level 2 predictor (neighbourhood affluence), controlling for the level 1 predictors. Non-linear (Bernoulli) analyses for a dichotomous outcome variable were used, along with the restricted penalised quasi-likelihood (PQL) method of estimation, which maximises the estimation of fixed effects. ${ }^{51}$ For the fixed effects, results reported are taken from the population average model with robust standard errors.

\section{RESULTS}

The initial unconditional model indicated that there was no significant between neighbourhood variation in body dissatisfaction $\left(\chi^{2}{ }_{(51)}=8.28, \mathrm{p}>0.5\right)$. In other words, nearly all the variation in our outcome variable was of the between person nature $(>99 \%)$ rather than of the between neighbourhood nature $(<1 \%)$. Therefore, body dissatisfaction was constrained to be fixed across neighbourhoods for subsequent analyses.

We first added age in years as a level 1 random effects predictor, centred around the grand mean. This enabled us to determine whether age was associated with body dissatisfaction in a way that differed across neighbourhoods. There was no significant random effect for age $\left(\chi^{2}{ }_{(51)}=47.05, \mathrm{p}<0.5\right)$, indicating that the relation between age and body dissatisfaction was constant across neighbourhoods. Thus, the effect of age was constrained to be fixed across neighbourhoods for subsequent analyses.

We next added individual body mass index as a level 1 random effects predictor, centred around the grand mean. This enabled us to determine whether body mass index predicted body dissatisfaction in a manner that differed across neighbourhoods, independent of age. Indeed, the effect of body mass index was random $\left(\chi^{2}{ }_{(51)}=84.44, p=0.002\right)$, indicating that the relation between body mass index and body dissatisfaction differed across neighbourhoods. At this step, we were also able to evaluate the role of age as an individual level fixed effect. We found that age was significantly associated with body dissatisfaction: $t(892)=-2.08, \mathrm{p}=0.038$, indicating that younger women were more likely to report body dissatisfaction.

To evaluate the role of individual affluence in predicting body dissatisfaction, independent of age and body mass index, we next added our dummy variable indicating "low income" $(<\$ 20000)$ versus "not low income" $(\geqslant \$ 20000)$ as a level 1 predictor with a random effect. We obtained a non-significant random effect for this variable $\left(\chi^{2}(51)=54.90, p=0.105\right)$, indicating that "individual affluence" predicts body dissatisfaction in a way that does not vary across neighbourhoods. Moreover, entered as a fixed effect, "individual affluence" also did not predict body dissatisfaction $(p=0.33)$. Thus, in our data, there is no evidence to suggest that body dissatisfaction covaries with individual affluence (whether low income or not). Despite these non-significant findings, we retained this dummy variable as a fixed effect in the model because we wanted to evaluate the effect of neighbourhood affluence, independent of individual affluence.

In the final step, we entered neighbourhood level affluence (average family income within the census tract), centred around the grand mean, as a level 2 predictor of the body mass index-body dissatisfaction relation. In other words, we evaluated whether "neighbourhood affluence" helped to explain the between neighbourhood variation in the body mass index-body dissatisfaction relation detected in an earlier step. 
Table 1 Results for hierarchical linear modelling analyses for evaluating the role of age, body mass index, individual affluence, and neighbourhood affluence on individual body dissatisfaction

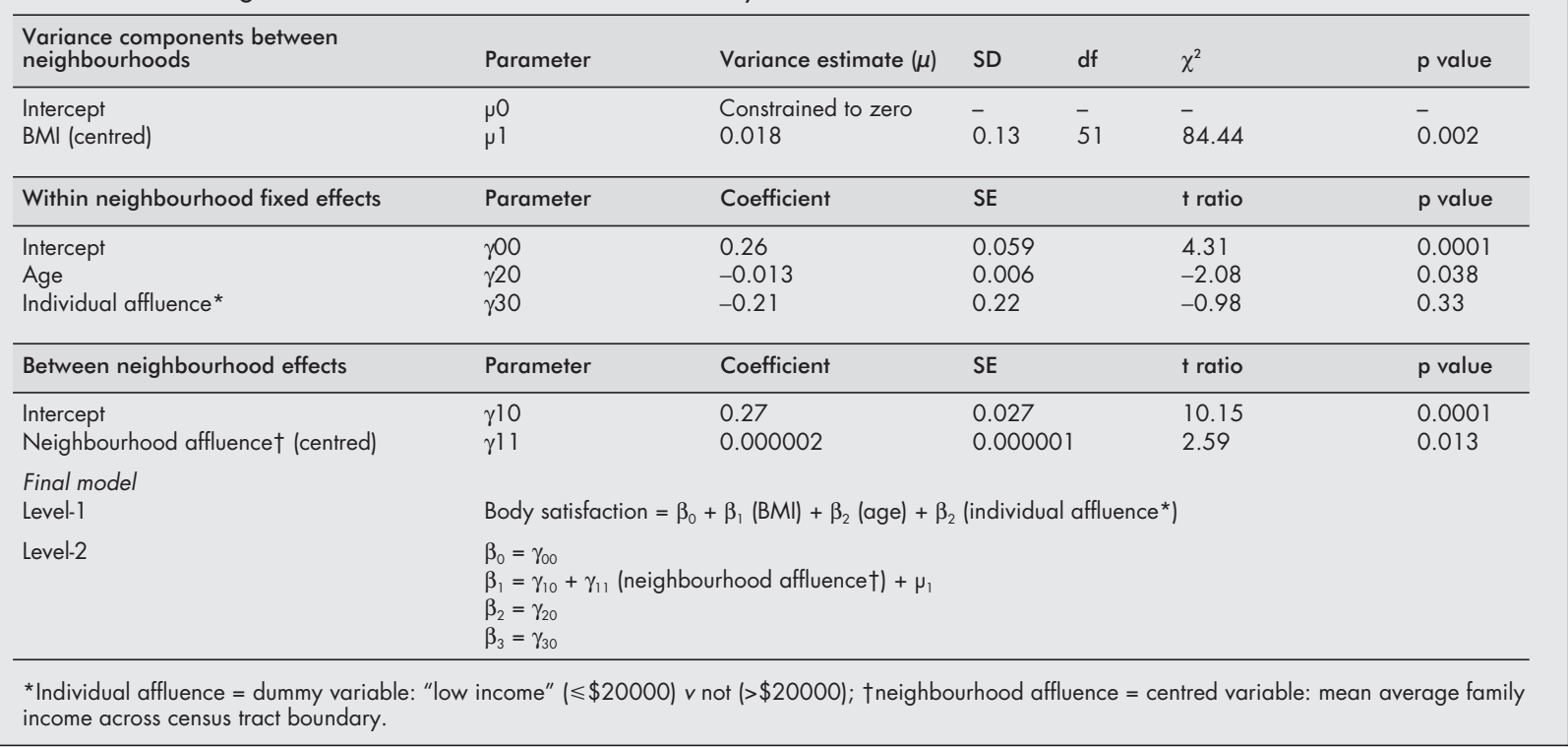

Results indicated that the variable body mass index-body dissatisfaction relation was indeed influenced by the affluence of the neighbourhood: $t(51)=2.59, \mathrm{p}=0.013)$. The direction of this relation was such that the more affluent the neighbourhood, the stronger the relation between body mass index and body dissatisfaction. Thus, for a given body mass index, a woman living in a neighbourhood of above average affluence was more likely to report body dissatisfaction than a woman living in a neighbourhood of average affluence or low affluence, and this was independent of whether she was low income or not at the individual level.f In other words, there was no evidence for an interaction between individual and neighbourhood levels of affluence: the impact of neighbourhood affluence on the body mass index-body dissatisfaction relation emerged regardless of the woman's individual affluence.

To illustrate this pattern of results, the following probabilities of body dissatisfaction, reported for a "non-low income" woman, were computed (recall that the proportion of body dissatisfaction across the entire sample was $53.4 \%$ ). A woman with an above average body mass index (approximately 28.6 $\mathrm{kg} / \mathrm{m}^{2}$, corresponding to the "overweight" category) had an $89 \%$ likelihood of reporting body dissatisfaction if she lived in a neighbourhood of above average affluence (approximate average income $\$ 90770$ ), versus an $82 \%$ likelihood of reporting body dissatisfaction if she lived in a neighbourhood of average affluence (approximate average income \$61 280), versus a $71 \%$ likelihood of reporting body dissatisfaction if she lived in a neighbourhood of below average affluence (approximate average income \$31 790). The values were more striking for a woman with an average body mass index (approximately 24 $\mathrm{kg} / \mathrm{m}^{2}$; "normal weight"): such a woman had a 71\% likelihood of reporting body dissatisfaction in a neighbourhood of above average affluence, a 58\% likelihood in a neighbourhood of average affluence, and a $43 \%$ likelihood in a neighbourhood of below average affluence. The highest probability of "body dissatisfaction" (among non-low income women) was found for a

$\ddagger$ We re-ran analyses using an alternative measure of body dissatisfaction for the outcome variable: discrepancy between ideal and actual weight. Results from these analyses were consistent with those reported in the text; namely, neighbourhood affluence emerged as a marginally significant predictor $(p=0.055)$ of a variable relation between body mass index and actual-ideal discrepancy.

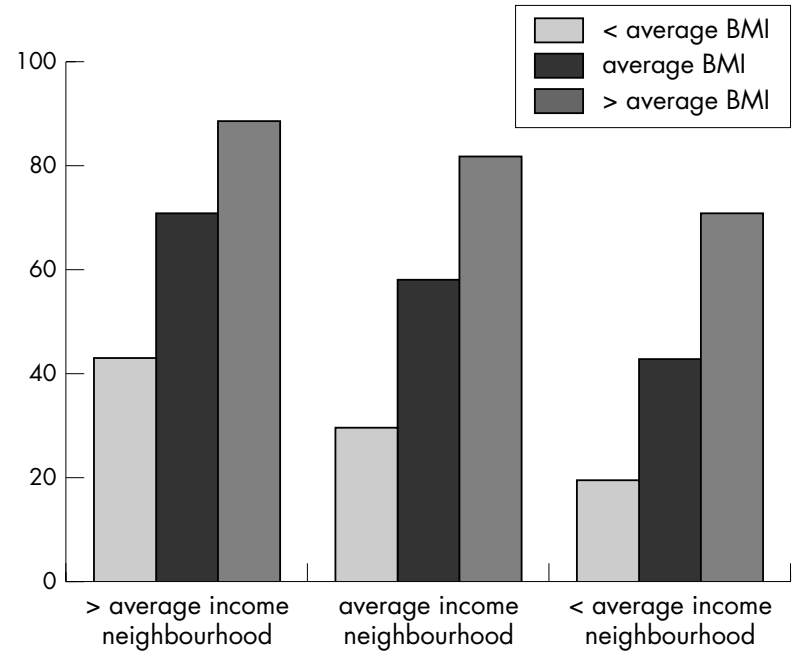

Figure 1 Proportion of women reporting body dissatisfaction by body mass index and neighbourhood affluence; reported for a non-low income woman (at least $\$ 20000$ annual income).

woman with an above average body mass index living in a neighbourhood of above average affluence (89\%); the lowest probability was found for a woman with a below average body mass index (approximately $19.5 \mathrm{~kg} / \mathrm{m}^{2}$, "underweight") living in a neighbourhood of below average affluence (19\%). Results are presented in table 1 , and illustrated in figure 1 .

The pattern of results obtained for "low income" women (not shown) was essentially identical except all proportion values were slightly $(1 \%-2 \%)$ lower.

\section{DISCUSSION}

Our main question pertained to the influence of neighbourhood level affluence on body dissatisfaction. Research on the relation between social class and eating disorders is mixed, and we proposed that contradictory findings reflect-in part-the failure of extant research to consider the affluence of a person's local environment as well as individual affluence. Our investigation encompassed the additional, broader goal of initiating a merge between two essentially discrepant literatures: the psychological perspective typically taken in research 
on body image, and the epidemiological and population health perspective that underlies the study of geographical variation in and socioeconomic determinants of health.

We found that although body dissatisfaction did not vary between neighbourhoods, the relation between body mass index and body dissatisfaction did show neighbourhood to neighbourhood variation-variation that reflected (in part) the level of affluence of the neighbourhood. For a given body mass index, a woman living in a highly affluent neighbourhood had a higher likelihood of reporting body dissatisfaction than a woman living in a neighbourhood of average affluence, who in turn had a higher likelihood of reporting body dissatisfaction than a woman living in a neighbourhood of low affluence. This finding was independent of the woman's individual affluence (whether she was low income or not).

Research on body dissatisfaction consistently implicates sociocultural standards of female attractiveness as an important macro level determinant of these phenomena. Although there remains little doubt regarding the existence and importance of these standards, such a position implies that these standards are ubiquitous. However, it is reasonable to suggest that the salience of this standard, as well as the degree to which it is pursued, varies across smaller living environments. Our results are consistent with this hypothesis. There is something about highly affluent neighbourhoods that makes body dissatisfaction more likely, and we suggest that the social climate in such neighbourhoods is one in which the values of the broader culture are magnified. For example, it is plausible that highly affluent neighbourhoods are characterised by a heightened sense of appearance investment, whereby people in general are more concerned about their body and appearance, and in which certain material features exist that facilitate investment in a person's appearance (for example, easy availability of fashion magazines depicting thin models, high number of weight loss centres or work out centres with a high emphasis on appearance, clothing stores that cater to a young, slim female population). Furthermore, perhaps the disadvantaged neighbourhoods show a lack of these features, and are thereby characterised by a social climate in which larger body sizes are accepted and body dissatisfaction is less common. This would help explain the higher prevalence of overweight in more disadvantaged areas. ${ }^{5}$ Additional research is needed to identify what it is about the affluence of a neighbourhood that translates into different prevalence values of body dissatisfaction.

To our knowledge, this study is the first to investigate variation in and determinants of body dissatisfaction at the neighbourhood level. One limitation of our data is their cross sectional nature. We cannot determine whether women experienced body dissatisfaction before living in their current neighbourhood, or whether it developed since having moved there, or whether body dissatisfaction or appearance investment influenced the type of neighbourhood she chose to live in. Greater understanding of these pathways would help us know whether local environmental affluence acts as a causal determinant of body dissatisfaction, or whether it acts to perpetuate existing body dissatisfaction, or both. An additional important limitation pertains to our indicator of individual level affluence. Because participants provided this information using ordinal categories (for example, less than $\$ 5000$, between $\$ 5000$ and $\$ 10000$, etc), it was necessary to use a rather rough measure of individual affluence (with dummy variables) to analyse this information. Furthermore, "income" is not synonymous with socioeconomic status; a composite measure including income, education, occupation, and material wealth may have more accurately categorised individuals in terms of social class.

None the less, our results provide initial support for the role of neighbourhood level determinants of body dissatisfaction. This finding should be replicated using a finer indicator of individual income and perhaps broader indicators of indi-

\section{Key points}

- Body dissatisfaction is common among women and can be associated with adverse psychological and behavioural consequences.

- Body dissatisfaction may be more common among high income women, although research results on this association are mixed.

- In this study we found that for a given body size (BMI), the likelihood of women's body dissatisfaction is greater if she lives in a more affluent neighbourhood.

vidual and neighbourhood social class (education, occupation, material deprivation). In addition, in line with existing and emerging neighbourhood level research, ${ }^{33}{ }^{52}$ future studies can begin the important task of identifying and examining pathways and mediating mechanisms of the relation between local environmental affluence and body dissatisfaction.

\section{Authors' affiliations}

L McLaren, L Gauvin, GRIS (Groupe de recherche interdisciplinaire en santé), University of Montreal, Canada

L Gauvin, Department of Social and Preventive Medicine, University of Montreal

\section{REFERENCES}

1 Gard MCE, Freeman CP. The dismantling of a myth: a review of eating disorders and socioeconomic status. Int J Eat Disord 1996;20:1-12.

2 Robert SA. Socioeconomic position and health: the independent contribution of community socioeconomic context. Annu Rev Sociol 1999;25:489-516.

3 Duncan C, Jones K, Moon G. Smoking and deprivation: are there neighbourhood effects? Soc Sci Med 1999;48:497-505.

4 Ecob R, Macintyre S. Small area variations in health related behaviours; do these depend on the behaviour itself, its measurement, or on personal characteristics? Health Place 2000;6:261-74

5 Ellaway A, Anderson A, Macintyre S. Does area of residence affect body size and shape? Int J Obes Relat Metab Disord 1997;21:304-8.

6 Kostanski M, Gullone E. Adolescent body image dissatisfaction: relationships with self-esteem, anxiety, and depression controlling for body mass. J Child Psychol Psychiatry 1998;39:255-62.

7 Allaz AF, Bernstein $M$, Rouget $P$, et al. Body weight preoccupation in middle-age and ageing women: a general population survey. Int J Eat Disord 1998;23:287-8.

8 Ledoux M, Rivard M. Poids corporel. Collection la santé et le bien-être: enquête sociale et de santé 1998. Québec: Institut de la statistique du Québec 2001:185-99.

9 Reboussin BA, Rejeski WJ, Martin KA, et al.Correlates of satisfaction with body function and body appearance in middle- and older aged adults: the Activity Counseling Trial (ACT). Psychol Health 2000;15:239-54

10 Fooken I. Sexuality in the later years - the impact of health and body-image in a sample of older women. Patient Educ Couns 1994;23:227-33.

11 Friedman MA, Dixon AE, Brownell KD, et al. Marital status, marital satisfaction, and body image dissatisfaction. Int J Eat Disord 1999;26:81-5

12 Ransdell LB, Wells CL, Manore MM, et al. Social physique anxiety in postmenopausal women. J Women Aging 1998;10:19-39.

13 King TK, Matacin M, Marcus BH, et al. Body image evaluations in women smokers. Addict Behav 2000;25:613-18.

14 Hunter MS, O'Dea I, Britten N. Decision-making and hormone replacement therapy: a qualitative analysis. Soc Sci Med 1997:45:1541-8.

15 Fauconnier A, Ringa V, Delanoë D, et al. Use of hormone replacement therapy: women's representations of menopause and beauty care practices. Maturitas 2000;35:215-28.

16 Steiger H, Séguin JR. Anorexia nervosa and bulimia nervosa. In: Millon T, Blaney PH, RD Davis. eds. Oxford textbook of psychopathology. New York: Oxford University Press, 1999:365-89.

17 Ogden J, Thomas D. The role of familial values in understanding the impact of social class on weight concern. Int J Eat Disord 1999;25:273-9.

18 Jeffery RW, French SA. Socioeconomic status and weight control practices among 20 - to 45 -year old women. Am J Public Health 1996;86:1005-10.

19 Drewnowski A, Kurth CL, Krahn DD. Body weight and dieting in adolescence: impact of socioeconomic status. Int J Eat Disord 1994:16:61-5.

20 Wardle J, Marsland L. Adolescent concerns about weight and eating; a social-developmental perspective. J Psychosom Res 1990;34:377-91. 
21 Story $M$, French SA, Resnick MD, et al. Ethnic/racial and socioeconomic differences in dieting behaviours and body image perceptions in adolescents. Int J Eat Disord 1995;18:173-9.

22 Eisler I, Szmukler GI. Social class as a confounding variable in the Eating Attitudes Test. J Psychiatr Res 1985:19:171-6.

23 Thompson SH, Sargent RG. Black and white women's weight-related attitudes and parental criticism of their childhood appearance. Women Health 2000;30:77-92

24 Stevens C, Tiggemann M. Women's body figure preferences across the life span. J Genet Psychol 1998;159:94-102.

25 Rogers L, Resnick MD, Mitchell JE, et al. The relationship between socioeconomic status and eating-disordered behaviours in a community sample of adolescent girls. Int J Eat Disord 1997;22:15-23.

26 Wakeling A. Epidemiology of anorexia nervosa. Psychiatry Res 1996:62:3-9.

27 Lee S. Reconsidering the status of anorexia nervosa as a western culture-bound syndrome. Soc Sci Med 1996;42:21-34.

28 Garner DM, Garfinkel PE, Schwartz D, et al. Cultural expectations of thinness in women. Psychol Rep 1980;47:483-91.

29 Katzmarzyk PT, Davis C. Thinness and body shape of Playboy centerfolds from 1978 to 1998. Int J Obes 2001;25:590-2.

30 Irving LM. Mirror images: effects of the standard of beauty on the selfand body-esteem of women exhibiting varying levels of bulimic symptoms. J Soc Clin Psychol 1990;9:230-42.

31 Field $\mathbf{A E}$, Cheung $\mathrm{L}$, Wolf $A M$, et al. Exposure to mass media and weight concerns among girls. Pediatrics 1999;103:E36-40.

32 Stice E, Schupak-Neuberg E, Shaw HE, et al. Relation of media exposure to eating disorder symptomatology: an examination of mediating mechanisms. J Abnorm Psychol 1994;103:836-40.

33 Leventhal T, Brooks-Gunn J. The neighbourhoods they live in: the effects of neighbourhood residence on child and adolescent outcomes. Psychol Bull 2000; 126:309-37.

34 Macintyre S, Ellaway A. Ecological approaches: rediscovering the role of the physical and social environment. In: Berkman LF, Kawachi I, eds. Social epidemiology. New York: Oxford University Press, 2000:332-48.

35 Sobal J, Stunkard AJ. Socioeconomic status and obesity: a review of the literature. Psychol Bull 1989;105:260-75.

36 Wilkinson RG. Unhealthy societies: the afflictions of inequality. London: Routledge, 1996
37 Wardle J, Griffith J. Socioeconomic status and weight control practices in British adults. J Epidemiol Community Health 2001;55:185-90.

38 O'Campo P, Xue X, Wang M-C, et al. Neighbourhood risk factors for low birthweight in Baltimore: a multilevel analysis. Am J Public Health 1997;87: 1113-18.

39 Evans RG, Barer ML, Marmor TR. Why are some people healthy and others not? New York: Aldine de Gruyter, 1994.

40 Marmot M, Wilkinson RG. Social determinants of health. Oxford: Oxford University Press, 1999

41 Bettle N, Bettle O, Neumärker U, et al. Adolescent ballet school students: their quest for body weight change. Psychopathology 1998:31:153-9.

42 Abraham S. Characteristics of eating disorders among young ballet dancers. Psychopathology 1996;29:223-9.

43 Garner DM, Garfinkel PE. Socio-cultural factors in the development of anorexia nervosa. Psychol Med 1980;10:647-56.

44 Garner DM, Garfinkel PE, Rockert W, et al. A prospective study of eating disturbances in the ballet. Psychother Psychosom 1987;48:170-5.

45 Gilmore J. Body mass index and health. Health Rep 1999;1 1:31-43.

46 Sampson RJ, Raudenbush SW, Earls F. Neighbourhoods and violent crime: a multilevel study of collective efficacy. Science 1997; 277:918-24.

47 Green KL, Cameron R, Polivy J, et al. Weight dissatisfaction and weight loss attempts among Canadian adults. Can Med Assoc J 1997;157| suppl 1):S17-25

48 Statistics Canada 1996 census [online]. Available from: URL: http://www.statisticscanada.ca/english/census96/list.htm

49 Rowland ML. Self-reported weight and height. Am J Clin Nutr 1990;52:1125-33.

50 Heymsfield SB, Allison DB, Heshka S, et al. Assessment of human body composition. In: Allison DB, ed. Handbook of assessment methods for eating behaviors and weight-related problems. New York: Sage, 1995:515-60.

51 Bryk AS, Raudenbush SW. Hierarchical linear models: applications and data analysis methods. Newbury Park, CA: Sage, 1992.

52 Elliott $M$. The stress process in neighbourhood context. Health Place 2000;6:287-99. 